\title{
Environmentally Friendly Polyvinyl alcohol-alginate/bentonite Nanocomposite Hydrogel Beads as Efficient Adsorbents for Removal of Toxic Methylene Blue from Aqueous Solution
}

\author{
Mona A. Aziz Aljar', Suad Rashdan ${ }^{1}$, Ahmed Abd El-Fattah ${ }^{12^{*}}$
}

\begin{abstract}
1Department of Chemistry, College of Science, University of Bahrain, P.O. Box 32038, Sakhir, Kingdom of Bahrain; maljar@uob.edu.bh ; srashdan@uob.edu.bh; aahussein@uob.edu.bh;

2Department of Materials Science, Institute of Graduate Studies and Research, Alexandria University, El-Shatby, 21526, Alexandria, Egypt; $\underline{\text { a abdelfattah@alexu.edu.eg; }}$

* Correspondence: Ahmed Abd El-Fattah, Ph.D Affiliation of the corresponding author: Department of Chemistry, College of Science, University of Bahrain, P.O. Box 32038, Sakhir, Kingdom of Bahrain; Department of Materials Science, Institute of Graduate Studies and Research, Alexandria University, El-Shatby, 21526, Alexandria, Egypt; aahussein@uob.edu.bh or a abdelfattah@alexu.edu.eg;

https://orcid.org/0000-0002-9282-8504
\end{abstract}

\begin{abstract}
Hazardous chemicals like toxic organic dyes are very harmful to the environment and their removal is quite challenging. Therefore there is a necessity to develop techniques, which are environment friendly, cost-effective and easily available in nature for water purification and remediation. The present research work is focused on the development and characterization of the ecofriendly polyvinyl alcohol (PVA) and alginate (Alg) hydrogel beads incorporating natural bentonite (Bent) clay as beneficial adsorbents for removal of toxic methylene blue (MB) from industrial water. PVA-Alg/Bent nanocomposite hydrogel beads with different Bent content $(0,10,20$, and $30 \mathrm{wt} \%$ ) were synthesized via external ionic gelation method. The designed porous and steady structure beads were characterized by the use of Fourier transform infrared spectroscopy (FTIR), energy-dispersive X-ray spectroscopy (EDX), and scanning electron microscopy (SEM). The performance of the beads as MB adsorbents was investigated by treating batch aqueous solutions. The experimental results indicated that the incorporation of Bent $(30 \mathrm{w} \%)$ in the nanocomposite formulation sustained porous structure, preserved water uptake, and increased MB removal efficiency by $230 \%$ compared to empty beads. Designed beads possessed higher affinity to MB at high $\mathrm{pH} 8,30^{\circ} \mathrm{C}$, and fitted well to pseudo-second-order kinetic model a high correlation coefficient. Moreover, designed beads had a good stability and reusability as they exhibited excellent removal efficiency $(90 \%)$ after six consecutive adsorption-desorption cycles. Adsorption process was found be combination of both monolayer adsorption on homogeneous surface and multilayer adsorption on heterogeneous surface. The maximum adsorption capacity of the designed beads system as calculated by Langmuir isotherm was found to be $51.34 \mathrm{mg} / \mathrm{g}$, which is in good agreement with the reported clay-related adsorbents. The designed PVA-Alg/Bent nanocomposite hydrogel beads demonstrated good adsorbent properties and could be potentially used for MB removal from polluted water.
\end{abstract}

Keywords: Alginate; polyvinyl alcohol; bentonite clay; nanocomposites hydrogel; cationic dyes; water remediation.

\section{Introduction}

Methylene blue (MB) is a well-known cationic phenothiazine dye, with versatile industrial application such as textile, leather, plastics, paper and cosmetics used as biologic stain and photosensitizer in photodynamic therapy [1-7]. However, MB is a non- 
biodegradable due to its complex aromatic structure contributing to a prolonged toxicity $[8,9,10]$. Consequently, it is necessary to remove toxic MB dye from industrial effluents and wastewater for a more secured and sustainable environment [11, 12].

Various conventional physicochemical techniques have been proposed and introduced for purification of water from toxic dyes including photocatalysis, chemical oxidation, ozonaization, membrane filtration, coagulation and flocculation, as well as adsorption [13-17]. Among all of these techniques, adsorption method is easily employed, prompt, and highly efficient $[18,19,20]$. Numerous different adsorbents have been synthesized such as activated carbons [21, 22], porous ceramics [23, 24], metal oxides [25], and polymeric hydrogels [26-30] for remediation of contaminated water. Indeed, from both environmental and industrial point of view, an ideal adsorbent should have large surface area, porous structure, available active functional sites, mechanical stability and strength, high strength as well as low cost and eco-friendly [31, 32, 33].

In recent years, the three-dimensional biodegradable polymeric hydrogels used as adsorbents have attracted wide attention [34]. During the removal process, the dye molecules can rapidly penetrate into the porous hydrogel networks in solution and therefore, combine with the hydrophilic active site groups like hydroxyl $(-\mathrm{OH})$ and carboxyl $(-\mathrm{COOH})$ that present on their surfaces $[26,35]$.

A striking candidate hydrogel for water purification is sodium alginate (Alg), a naturally occurring anionic biopolymer, obtained from brown algae. It has unique properties, including low cost, biodegradability, biocompatibility, and flexibility in hydrated environment. In addition, the ionized negatively-charged carboxylate(-COO-) groups of Alg are able to attract cationic MB from aqueous solutions [36, 37, 38]. Nevertheless, Alg hydrogel beads usually give poor mechanical strength. To improve this property, Alg can be blended with polyvinyl alcohol (PVA), which possesses desirable properties such as non-toxicity, biodegradability, high elasticity, and mechanical strength [39]. Despite that, several researchers reported that PVA-Alg hydrogel has limited application in dye removal from wastewater owing to its relatively low adsorption capacity and selectivity $[37,38,40]$.

Nanoparticles based on clay minerals, have extensively received great attention due to their remarkably low-cost, large abundance, high specific surface area, and good intrinsic adsorption characteristics [11, 23, 31]. Because of the anionic nature of the clay nanoparticles in aqueous media, a number of studies have been reported the efficacy of clay minerals for removing cationic dyes from waste water [41, 42]. Bentonite (Bent) is a naturally occurring clay that is composed tetrahedral Si layer and octahedral Al layer which are interlinked and containing reactive $-\mathrm{OH}$ groups. The charged layers are neutralized by the presence of cations like $\mathrm{Na}+$ and $\mathrm{K}+$ and the interlayer space holding some amount of water [43]. Owing to these exciting features, incorporating Bent nanoparticles into the PVA-Alg hydrogel matrices to form nanocomposite hydrogels can significantly improve adsorption performance and further increase the mechanical stability of the hydrogel adsorbents [44, 45].

The objective of the present research is to continue to explore the advantages of both ecofriendly polymeric hydrogel and natural nanoparticle-based materials. In particular, our aim is to synthesize and characterize biodegradable nanocomposite, with enhanced mechanical stability and adsorption properties as well as understanding the adsorption mechanism in order to assess the best commercial formulations for industrial applications.

In this work, PVA-Alg/Bent nanocomposite hydrogel beads were prepared via external ionic gelation method and applied to the removal of toxic MB dye from aqueous 
solution. The structure, composition, morphology, and swelling of the assembled beads were investigated. Many factors affecting the MB adsorption from the aqueous solution by PVA-Alg/Bent nanocomposite hydrogel beads were studied in detail, including different Bent contents, $\mathrm{pH}$ values, contact time, initial MB concentration, different adsorbent dosage, temperature, and reusability. Further, adsorption kinetics, adsorption isotherms, and adsorption mechanism were analyzed.

\section{Materials and methods or Experimental}

\subsection{Materials}

Polyvinyl alcohol (PVA; molecular weight: 13000-23000 g/mol; 89 mol\% hydrolyzed), low viscosity alginate (Alg) powder, calcium chloride $\left(\mathrm{CaCl}_{2}\right)$, hydrochloric acid $(\mathrm{HCl})$, sodium hydroxide $(\mathrm{NaOH})$, and methylene blue ( $\mathrm{MB}$; chemical formula: $\mathrm{C}_{16} \mathrm{H}_{18} \mathrm{~N}_{3} \mathrm{SCl}$, molecular weight: $319.85 \mathrm{~g} / \mathrm{mol}$, solubility in water: $40 \mathrm{~g} / \mathrm{L}, \lambda \mathrm{max}=663 \mathrm{~nm}$, as shown in Figure 1a) were purchased from Sigma-Aldrich Co., Germany and used without further purification. Bentonite (Bent) clay nanoparticles (Bent; molecular weight: $180.1 \mathrm{~g} / \mathrm{mol}$; chemical formula: $\mathrm{H}_{2} \mathrm{Al}_{2} \mathrm{O}_{6} \mathrm{Si}$;), with average diameter of $24.81 \pm 1.86 \mathrm{~nm}$ (Figure 1b) was obtained as a gift from Sphinx Milling Station Co., Alexandria, Egypt. The deionized water $(0.055 \mu \mathrm{S} / \mathrm{cm})$ was used in all experiments.
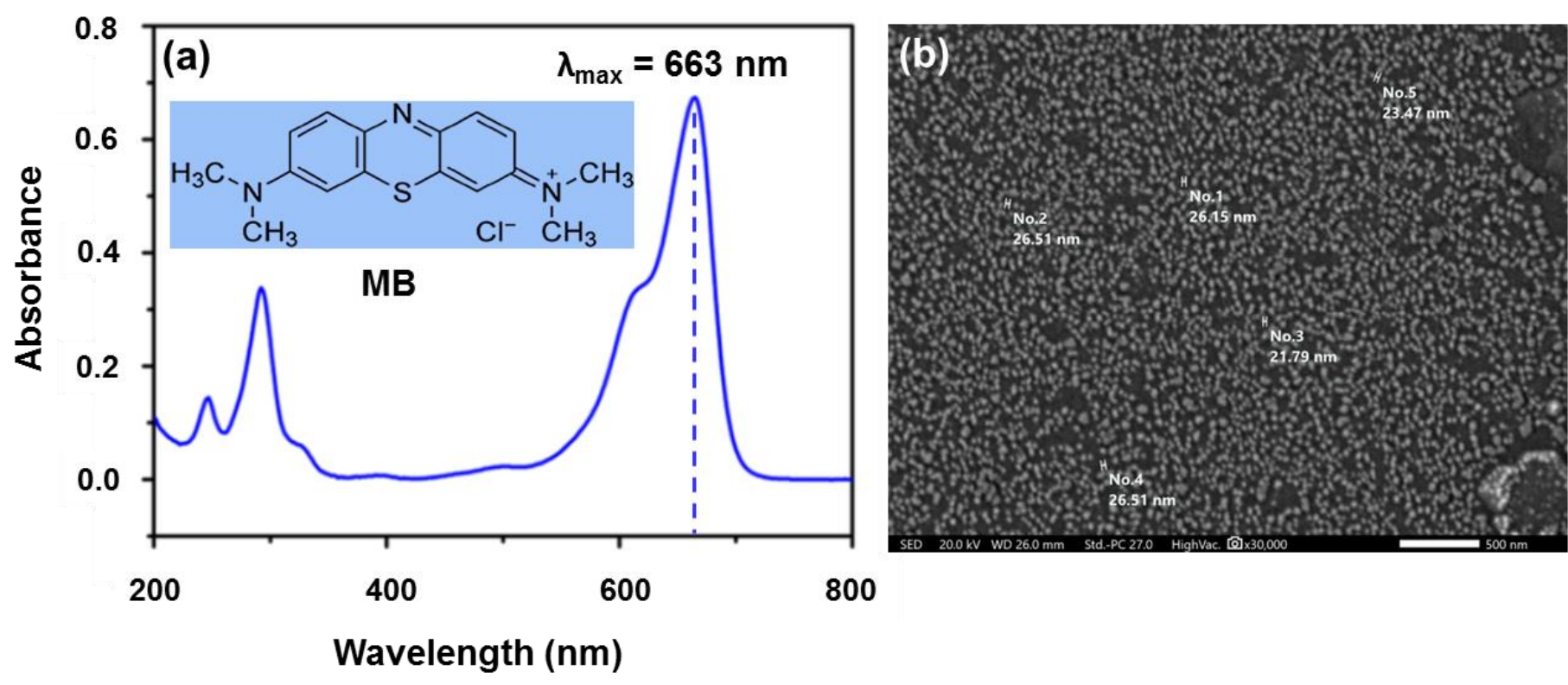

Figure 1. (a) UV/Vis spectrum of $M B$ dye (the inset shows the chemical structure of $M B$ ) and (b) SEM micrograph of bentonite clay nanoparticles.

\subsection{Preparation of Nanocomposite Hydrogel Beads}

PVA (2 g) and Alg (2 g) were dissolved in deionized water (100 mL) in a water bath at $90 \mathrm{oC}$ under constant stirring at $350 \mathrm{rpm}$ for $2 \mathrm{~h}$ to achieve a homogeneous solution. After complete dissolving, the solution was stirred for an additional $1 \mathrm{~h}$ and cooled to room temperature. Then, a predetermined content of the Bent powder (10, 20 and $30 \mathrm{wt} \%$ in relation to the polymers) was dispersed in the aqueous PVA-Alg solution via sonication at $40{ }^{\circ} \mathrm{C}$ for $30 \mathrm{~min}$, and the mixture was vigorously stirred for $1 \mathrm{~h}$ in a shaker. The obtained viscous suspension was slowly added dropwise by a syringe into the stirring $\mathrm{CaCl}_{2}$ solution $(3 \%, 600 \mathrm{~mL})$, and the nanocomposite hydrogel beads were obtained. To complete the gelation reaction, the beads were maintained in the $\mathrm{CaCl}_{2}$ solution overnight. Finally, the beads were filtered, washed three times with deionized water to remove any unreacted $\mathrm{CaCl}_{2}$, dried for $48 \mathrm{~h}$ at room temperature, and stored in a clean 
glass bottle for further characterization and adsorption study. Control hydrogel beads without Bent clay were prepared using the same previous steps.

\subsection{Characterization Methods}

\subsubsection{Fourier Transform Infrared Spectroscopy (FTIR)}

Chemical compositions of the Bent, PVA-Alg control hydrogel and PVA-Alg/Bent nanocomposite hydrogel were analyzed by FTIR spectroscopy with the FT-IR spectrometer Spectrum Two (Perkin-Elmer, Waltham, MA, USA). The spectrum was recorded throughout the wavenumber range from 4000 to $400 \mathrm{~cm}-1$, at a resolution of $4 \mathrm{~cm}-1$ with 32 scans per sample. The sample was prepared using the $\mathrm{KBr}$ disc technique in which the sample $(5 \mathrm{mg})$ was compressed with $\mathrm{KBr}$ powder $(200 \mathrm{mg})$ by hydraulic pressure to form a disk with a diameter of $12 \mathrm{~mm}$.

\subsubsection{Scanning Electron Microscopy (SEM)}

A scanning electron microscope (SEM) was used to assess the surface morphology of the desired beads. For this purpose, SEM uses a JEOL instrument (JSM-5300, Tokyo, Japan), which was operated at $25 \mathrm{keV}$. Prior to SEM imaging, the samples were ultrasonically washed for $30 \mathrm{~s}$ and sputter-coated with gold to a thickness of $0.04 \mu \mathrm{m}$ in a sputter-coating unit (JFC 1100 E, Tokyo, Japan).

\subsubsection{Energy-Dispersive X-ray Spectroscopy (EDX)}

Elemental composition of the Bent, PVA-Alg control hydrogel and PVA-Alg/Bent nanocomposite hydrogel was determined using energy dispersive X-ray (EDX) microanalysis attached to the SEM. Analysis was performed on uncoated samples at $15 \mathrm{kV}$ for $60 \mathrm{~s}$.

\subsubsection{Swelling Behavior}

To estimate the swelling behavior of the beads, the dried beads were immersed in deionized water $(500 \mathrm{~mL})$ at different $\mathrm{pH}$ values $(3,7$, and 9$)$ for $5 \mathrm{~h}$ at room temperature until the beads reached equilibrium swelling. During this experiment, water was occasionally exchanged several times. The swollen beads were taken out of the aqueous solution at fixed time scheduled, surface wiped and weighted to obtain their wet weights $\left(\mathrm{W}_{\mathrm{w}}\right)$. Then they were dried at $40^{\circ} \mathrm{C}$ in an oven until constant weight was achieved. After which, they were weighted again to determine their dry weights $\left(\mathrm{W}_{\mathrm{d}}\right)$. The average of five measurements was taken as the final result. The percentage of swelling ratio by each sample was calculated according to Equation (1) [46].

\section{Swelling ratio $(\%)=\left[\left(\mathrm{W}_{\mathrm{w}}-\mathrm{W}_{\mathrm{d}}\right) / \mathrm{W}_{\mathrm{d}}\right] \times 100$}

\section{4. $M B$ Adsorption Experiments}

The adsorption of MB from aqueous solution onto PVA-Alg/Bent nanocomposite hydrogel beads was systematically estimated. Before adsorption experiment, a stock solution of MB (500 ppm) was prepared by dissolving accurately weighed amount of $\mathrm{MB}$ powder in deionized water. The prepared stock solution was diluted to various $M B$ concentrations of the same $\mathrm{pH}$ and absorbance was measured by using UV/Vis spectrophotometer (Lambda 35, Perkin-Elmer, Waltham, MA, USA) which records characteristic absorption peak of $\mathrm{MB}$ at wavelength of $663 \mathrm{~nm}$. A calibration curve was constructed with reference to absorbance values of corresponding concentration of MB. The calibration curve equation was used to find the concentration of MB before and after adsorption. The adsorption experiments were studied by batch adsorption technique in which a certain amount of dried beads was added to a $100 \mathrm{~mL}$ Erlenmeyer flask containing MB solution $(50 \mathrm{~mL})$ of a certain concentration and placed in a mechanical water bath shaker 
at $150 \mathrm{rpm}$ for a predetermined period of time. Then, the supernatant was centrifuged and the concentration was measured by UV/Vis spectrophotometer. Effect of different Bent content $(10,20$, and $30 \mathrm{wt} \%)$, different $\mathrm{pH}(2-10)$, contact time (20-320 min), initial MB concentration (50-350 ppm), PVA-Alg/Bent dosage (0.1-2 g), and temperature (20, 30,40 , and $50{ }^{\circ} \mathrm{C}$ ) have been explored to evaluate the performance of adsorbent beads. The equilibrium adsorption capacity, qe, $(\mathrm{mg} / \mathrm{g})$ of $\mathrm{MB}$ onto adsorbent beads as well as the removal percentage (\%) were calculated from Equations (2) [47] and (3) [48, 49] respectively.

$$
\begin{aligned}
& \mathrm{q}_{\mathrm{e}}=\left(\mathrm{C}_{0}-\mathrm{C}_{\mathrm{e}}\right) \times \mathrm{V} / \mathrm{m} \\
& \operatorname{Removal}(\%)=\left[\left(\mathrm{C}_{0}-\mathrm{C}_{\mathrm{e}}\right) / \mathrm{m}\right] \times 100
\end{aligned}
$$

where $\mathrm{Co}$ and $\mathrm{Ce}$ are the initial and equilibrium concentrations of $\mathrm{MB}(\mathrm{mg} / \mathrm{L})$, respectively, $\mathrm{m}$ is the adsorbent mass $(\mathrm{g})$ and $\mathrm{V}$ is the MB solution volume (L).

\subsection{Adsorbent Reusability Study}

The reusability of PVA-Alg/Bent adsorbent beads was carried out in the repeating adsorption-desorption process. PVA-Alg/Bent beads after adsorbing MB were washed thoroughly with deionized water and dried for $48 \mathrm{~h}$ at room temperature. Then, the beads were placed in a glass bottle containing $\mathrm{HCl}$ solution $(0.1 \mathrm{M}, 50 \mathrm{~mL})$ and shaked at $150 \mathrm{rpm}$ for $30 \mathrm{~min}$ for the regeneration of the beads. The regenerated beads were separated from the solution, rinsed by deionized water till no more MB leached out and reused to conduct the same adsorption procedure at the same conditions. Six adsorptiondesorption cycle tests, were carried out.

\subsection{Adsorbent Isotherms}

The adsorption mechanisms were studied by Freundlich and Langmuir isotherm models. The Freundlich isotherm adsorption formula is expressed in Equation (4) [50, 51].

$$
\ln q_{e}=\ln K_{F}+\frac{1}{n} \ln C_{e}
$$

where $K_{F}\left[(\mathrm{mg} / \mathrm{g})(\mathrm{L} / \mathrm{mg})^{1 / \mathrm{n}}\right]$ and $\mathrm{n}$ are the Freundlich constants, which represent the adsorption capacity and adsorption intensity, respectively. For favorable and multilayer cooperative adsorption, $1 / \mathrm{n}$ value should be between 0 and 1 [51].

The Langmuir isotherm adsorption Equations (5) and (6) $[50,51]$ were defined as follows:

$$
\begin{gathered}
\frac{C_{e}}{q_{e}}=\frac{1}{K_{L} q_{m}}+\frac{C_{e}}{q_{m}} \\
R_{L}=\frac{1}{1+K_{L} C_{o}}
\end{gathered}
$$

Where $\mathrm{q}_{\mathrm{m}}(\mathrm{mg} / \mathrm{g})$ is the maximum adsorption capacity, $\mathrm{K}_{\mathrm{L}}(\mathrm{L} / \mathrm{mg})$ is the Langmuir constant related to the affinity of the binding sites, and RL is the constant separation factor. $R_{L}$ value between 0 and 1 states a favorable adsorption process [50].

\subsection{Adsorbent Kinetics}

Both pseudo-first and pseudo-second order kinetic models were adopted to estimate the adsorption kinetics of $\mathrm{MB}$ dye onto PVA-Alg/Bent nanocomposite hydrogel beads. The corresponding Equations $(7,8)[50,51]$ displayed in their linear forms are as follows:

$$
\ln \left(q_{e}-q_{t}\right)=\ln q_{e}-K_{1} t
$$




$$
\frac{\mathrm{t}}{q_{t}}=\frac{1}{K_{2} q_{e}^{2}}+\frac{1}{q_{e}} t
$$

where $\mathrm{q}_{\mathrm{e}}$ and $\mathrm{q}_{\mathrm{t}}(\mathrm{mg} / \mathrm{g})$ are the amounts of $\mathrm{MB}$ adsorbed at equilibrium and at time $\mathrm{t}$, respectively. $\mathrm{K}_{1}(1 / \mathrm{min})$ and $\mathrm{K}_{2}(\mathrm{~g} / \mathrm{mg}$.min) are the rate constants of pseudo-first order and pseudo-second-order kinetics respectively.

\subsection{Adsorbent Kinetics}

The significant differences between the data among the experimental groups were analyzed using a one-way ANOVA test followed by Turkey's Post Hoc test and student t-test. All data were represented as mean \pm standard deviation $(M \pm S D)$ and each experiment was performed at least five replicates. A $p$ value $<0.05$ was considered significant.

\section{Results and discussion}

\subsection{Fabrication of Nanocomposite Hydrogel Beads}

The PVA-Alg control hydrogel and PVA-Alg/Bent nanocomposite hydrogel beads were successfully prepared by external ionic gelation method with $\mathrm{Ca}^{2+}$ ions as crosslinkers [44, 47]. Figures $2 \mathrm{a}$ and $2 \mathrm{~b}$ shows the digital images of the wet and dry PVA-Alg/Bent beads as well as the dry beads after adsorption of MB. The incorporation of Bent nanoparticles into the hydrogel beads was confirmed by the apparent beige color of the beads. The wet beads exhibited uniformly spherical shape with a smooth surface and had a size of approximately $4 \mathrm{~mm}$ (Figure 2a). However, during air drying, the initial spherical shape of the beads was slightly changed, resulting in rough surface morphology without a marked collapse on the surface, indicating an improved mechanical stability (Figure 2b). Indeed, the deformation in shape is inevitable when water was evaporated from the wet hydrogel beads throughout the drying process, causing volume shrinkage of hydrogel beads [52]. Figure 2c reveals black color of the beads indicated encapsulation of $\mathrm{MB}$ in the nanocomposite hydrogel beads.

In this work, the PVA: Alg weight ratio and the content of Bent nanoparticles were fixed at (1:1) and $30 \mathrm{wt} \%$ respectively. These values were selected because they represent an optimum performance of efficient adsorptive removal of MB dye and mechanical stability of the hydrogel beads [44]. To assess the adsorption ability of the designed nanocomposite hydrogel beads, preliminary adsorption tests were performed. As shown in Figure $2 \mathrm{~d}$, the control hydrogel beads had limited removal rate (28.53\%) for MB. Nevertheless, the removal rate of $\mathrm{MB}$ was significantly improved by increasing the content of Bent nanoparticles in the hydrogel beads. More specifically, the incorporation of $10 \mathrm{wt} \%$, $20 \mathrm{wt} \%$, and $30 \mathrm{wt} \%$ Bent nanoparticles in the hydrogel beads increased the removal rate of MB dye by $165 \%, 190 \%$, and $230 \%$ respectively compared to the control hydrogel beads. However, Bent contents exceeding the selected value were denounced to avoid aggregation of Bent nanoparticles, which in turn may deteriorate the overall mechanical stability and beads strength $[53,54]$. After taking the factors of adsorption amount and mechanical strength into consideration, PVA-Alg/Bent (30 wt\%) nanocomposite hydrogel with appropriate MB removal percentage (94.64\%) and stiffness was selected for subsequent characterizations and adsorption study.

\subsection{Structure and Chemical Composition}

Figure 3 displays the FTIR spectra of the pristine Bent, PVA-Alg control hydrogel, and PVA-Alg/Bent nanocomposite hydrogel. Bent exhibits characteristic absorption peaks at $1054,982,3674$, and $835 \mathrm{~cm}-1$. Absorption peak at $1054 \mathrm{~cm}-1$ is attributed to the stretching vibrations of Si-O-Si group, whereas the peak at $950 \mathrm{~cm}-1$ is related to $\mathrm{Al}-\mathrm{OH}-\mathrm{Al}$ bending vibrations [50,53]. Besides, very weak bands at 3674 and $835 \mathrm{~cm}-1$ 
are corresponding to $\mathrm{O}-\mathrm{H}$ and $\mathrm{Si}-\mathrm{O}$ stretching vibrations of silanol group of bent. The spectrum of the PVA-Alg hydrogel, shows a broad overlapped absorption band in the region of $3300-3400 \mathrm{~cm}-1$ which is related the stretching vibration of $\mathrm{O}-\mathrm{H}$ of the PVA and Alg [44, 47]. Absorption peaks in the area of $1635 \mathrm{~cm}-1$ and $1428 \mathrm{~cm}-1$ are due to asymmetric and symmetrical stretching vibrations of the $-\mathrm{COO}$ of $\mathrm{Alg}$ [44,55]. The peak appeared at $2947 \mathrm{~cm}-1$ was the typical stretching vibration for $\mathrm{C}-\mathrm{H}$ in PVA. All the characteristic peaks of PVA, Alg, and Bent were observed in the FTIR spectrum of the nanocomposite hydrogel. In addition, the small shift in the position of the band near 1300 $\mathrm{cm}-1$ would be related to the presence of hydrogen bonds between $\mathrm{O}-\mathrm{H}$ groups of PVA and Alg or with Si-O groups of Bent $[44,53,55]$. These results suggested that the Bent clay had successfully incorporated in the PVA-Alg hydrogel polymer matrix.
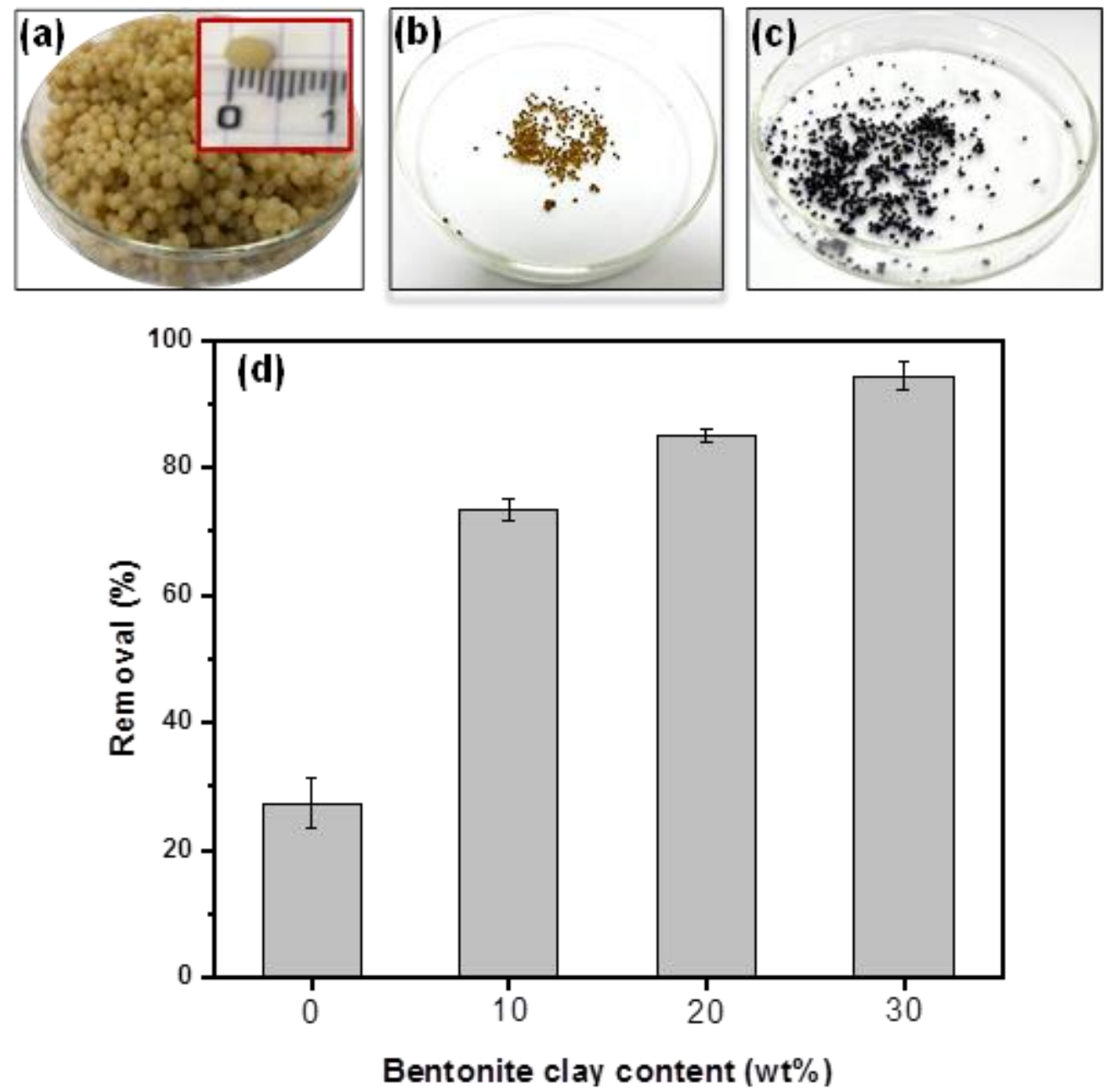

Figure 2. PVA-Alg/Bent nanocomposite hydrogel beads (a) before drying (inset shows the size of wet beads), (b) after $24 \mathrm{~h}$ air drying, (c) after adsorption of MB dye, and (d) effect of bentonite content $(0,10,20,30 \mathrm{wt} \%)$ on the removal percentage of $\mathrm{MB}\left(\mathrm{C}_{\mathrm{o}}[\mathrm{MB}]=\right.$ $200 \mathrm{mg} / \mathrm{L}, \mathrm{V}=50 \mathrm{~mL}, \mathrm{pH}=8$, beads dosage $=1.5 \mathrm{~g}, \mathrm{t}=300 \mathrm{~min}, \mathrm{~T}=30^{\circ} \mathrm{C}$ ).

The presence of Bent nanoparticles homogeneously distributed in the hydrogel matrix was further confirmed by EDX elemental analysis as shown in Figure 4. EDX spectrum of Bent clay (Figure 5a) confirmed the presence of its main constituents such as $\mathrm{O}$ $(51.70 \%), \mathrm{Si}(21.89 \%), \mathrm{Al}(9.81 \%), \mathrm{Fe}(6.88 \%)$, and C $(4.61 \%)[50,56]$. The main characteristic peaks attributed to $\mathrm{C}(40.95 \%)$ and $\mathrm{O}(52.19 \%)$ elements are detected in the EDX spectrum of control hydrogel (Figure $4 \mathrm{~b}$ ), which are due to the contribution of PVA and 
Alg component. It was worth noting that the peaks of $\mathrm{Si}, \mathrm{Al}$ and $\mathrm{Fe}$ elements can be clearly observed in the EDX spectrum (Figure 4c) of PVA-Alg/Bent nanocomposite hydrogel, indicated the successful incorporation of Bent nanoparticles on the hydrogel.

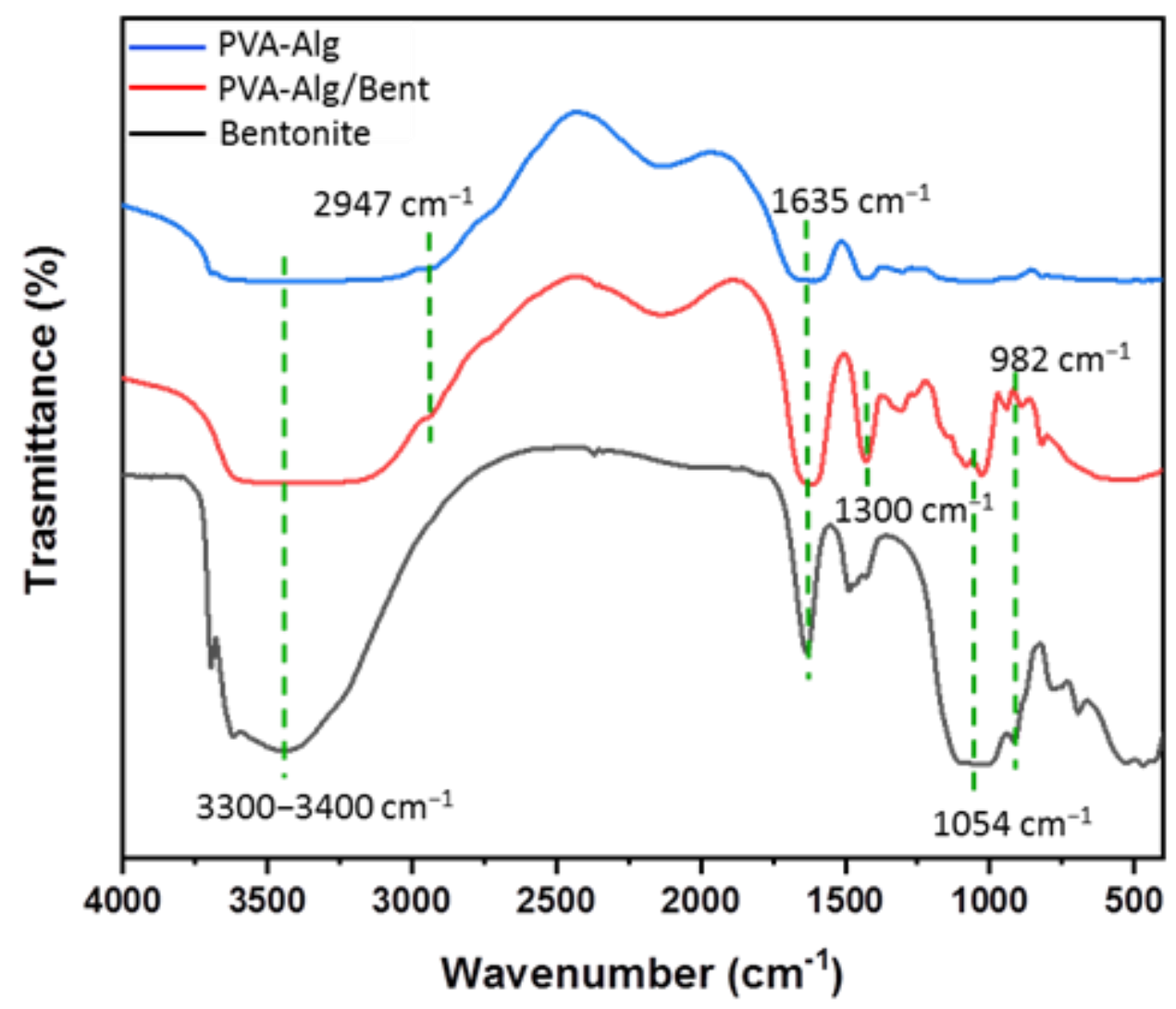

Figure 3. FTIR spectra of pristine Bent, PVA-Alg hydrogel and PVA-Alg/Bent nanocomposite hydrogel.

\subsection{Morphological observation}

Figure 6 presents the SEM micrographs of the dried entire beads and their surfaces for both control and nanocomposite hydrogels. SEM micrographs revealed a slight increase in the size of the nanocomposite bead (Figure 5a) compared to the unfilled bead (Figure 5c). Besides, both beads exhibited an irregular gully shape with some cracks and pores on the surface. However, as displayed in Figures $5 b, d$, the incorporation of Bent (30 $\mathrm{wt} \%)$ in the hydrogel resulted in a clear decrease in the size of the pores and the thickness of the cracks . Furthermore, the nanocomposite hydrogel showed uniform dispersion of the clay nanoparticles within the polymer matrix, leaving a relatively rough surface (Figure $5 \mathrm{~d}$ ). This structure will provide more contact sites and convenient diffusion channels, which may contribute to a better MB dye adsorption by PVA-Alg/Bent beads $[44,52,56]$. 

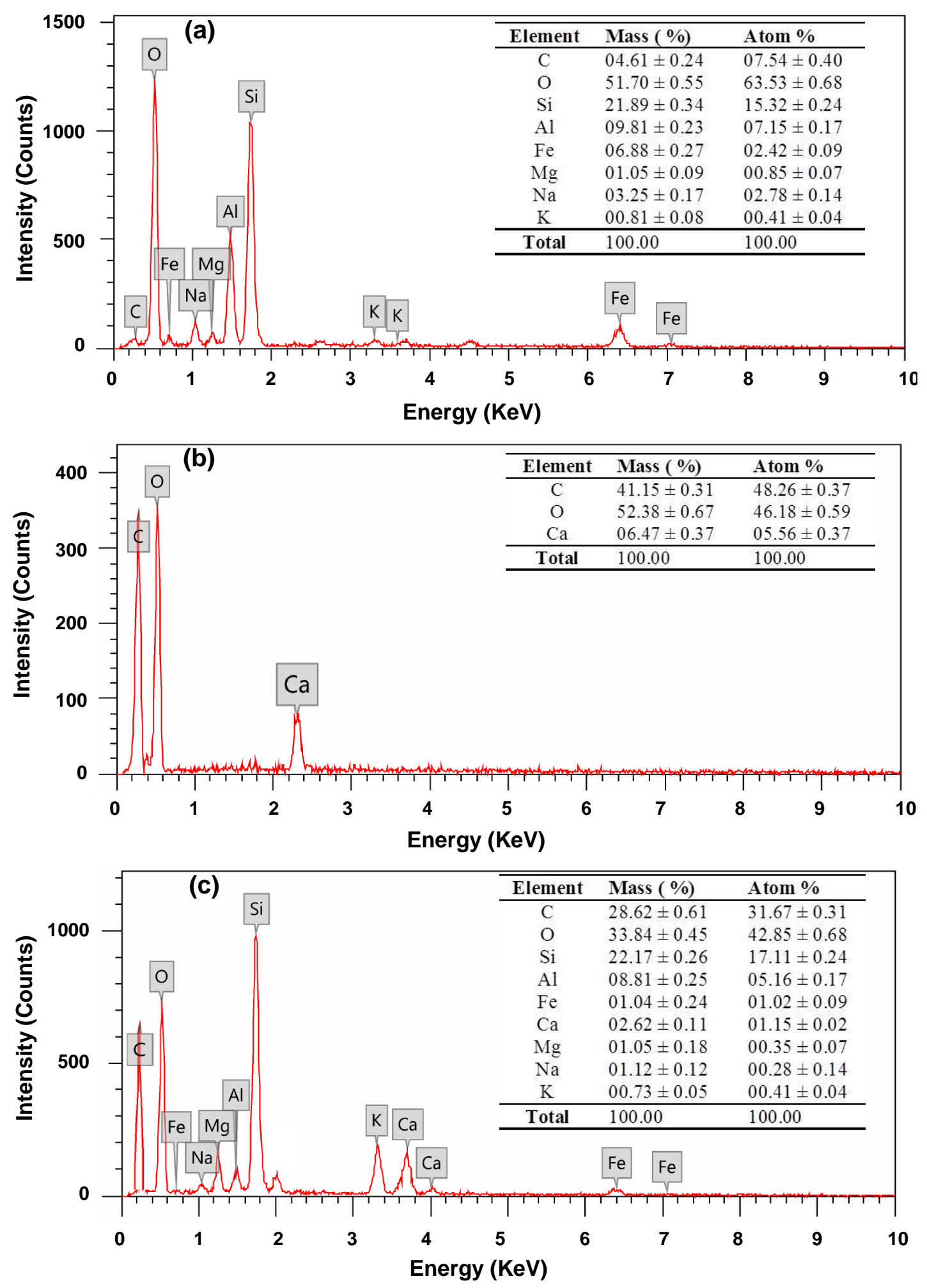

Figure 4. EDX spectra of (a) pristine bentonite clay (b) PVA-Alg control hydrogel, and (c) PVA-Alg/Bent nanocomposite hydrogel. The insets show the corresponding weight and atomic percentages of the elements present in the samples 

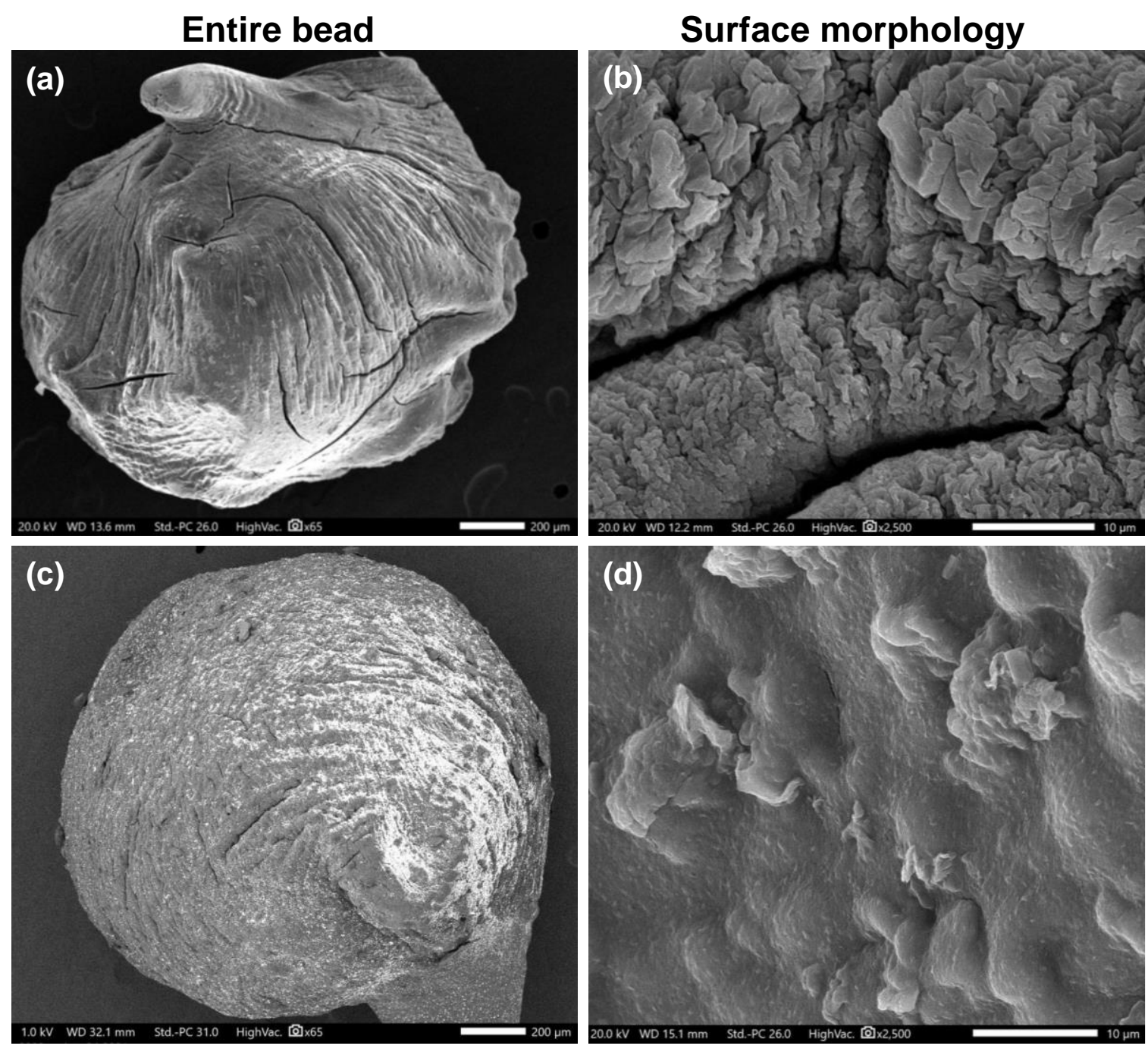

Figure 5. SEM micrographs of (a, b) PVA-Alg control hydrogel beads, and (c, d) PVA-Alg/Bent nanocomposite hydrogel beads.

\subsection{Swelling Behavior}

The swelling behavior of PVA-Alg/Bent (30 wt\%) nanocomposite hydrogel beads in aqueous solution at different $\mathrm{pH}$ values is depicted in Figure 6. Clearly, all beads showed a similar tendency with different degrees of hydration. As can be seen from Figure 6a, the swelling ratio enhanced slightly during the initial swelling stage (20-150 $\mathrm{min})$, and then turn into slower until reaching a plateau (150-320 min). One reasonable explanation for this observation was attributed to the presence of $-\mathrm{OH}$ groups on the polymer chains and clay surfaces that attract water molecules from the soaking medium, promoting their penetration into the gel-network, and eventually, elevating water uptake ability of the nanocomposite beads [56, 57]. Moreover, hydrogel beads exhibited obvious $\mathrm{pH}$-dependent swelling behavior, where, the water uptake of the beads increased significantly with increasing $\mathrm{pH}$ of the solution. More specifically, at $150 \mathrm{~min}$, the swelling rate increased gently within $\mathrm{pH} 3-7$ (from $25.5-30.2 \%$ ), and increased speedily in the range of $\mathrm{pH} 7-9$ (from 30.2-40.6\%) indicating that the structure of the cross-linked hydrogel changed at different $\mathrm{pH}$ values (Figure 6b). The low swelling capacity at acidic solution ( $\mathrm{pH} 3$ ) can be due to the protonation of $-\mathrm{COO}^{-}$to $-\mathrm{COOH}$ groups of the hydrogel matrix. Consequently, the beads existed in collapsed state via the hydrophobic interaction, lead to more rigid gel-networks structure, which restricted traversing of water [44,51, 57]. In neutral and alkaline solution ( $\mathrm{pH} 9$ ), some of the $-\mathrm{COOH}$ groups in the hydrogel ionized 
to - $\mathrm{COO}^{-}$, which establishing repulsions between the negatively charged chains, facilitating the expansion of hydrogel and thus enhancing the swelling rate $[51,57]$.
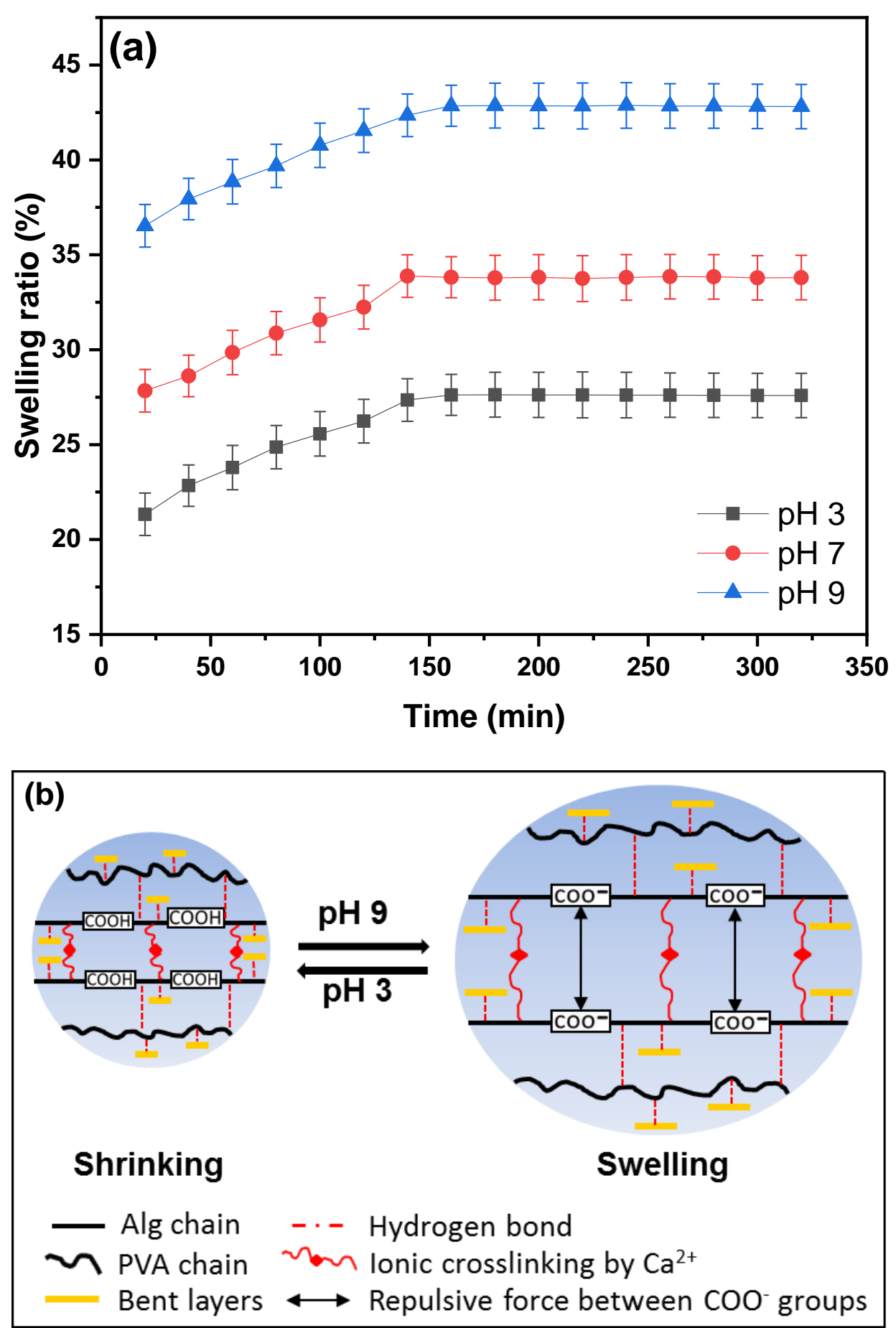

Figure 6. Swelling behavior of PVA-Alg/Bent nanocomposite hydrogel beads at different $\mathrm{pH}$ values: (a) swelling ratio and (b) Schematic illustration 
To study the suitability of the designed PVA-Alg/Bent (30 wt\%) nanocomposite hydrogel beads as adsorbent for MB dye, adsorption experiments were first conducted utilizing a batch experimental setup. The influences of various parameters on adsorption behaviors of the beads, such as $\mathrm{pH}$, contact time, initial dye concentration, beads dosage and temperature, were explored.

\subsubsection{Effect of Solution $\mathrm{pH}$}

The $\mathrm{pH}$ of dye solution is the most vital parameter controlling the efficiency of dye adsorption process [57]. The effect of solution $\mathrm{pH}$ on the adsorption capacity and removal percentage of MB dye by PVA-Alg/Bent hydrogel is illustrated in Figure 7a. As the $\mathrm{pH}$ increased from 2 to 5 , the adsorption capacity of MB was low and kept roughly constant, however, raised significantly within the $\mathrm{pH}$ range from 5 to 8 , and finally decreased at $\mathrm{pH}$ values greater than 8 . Maximum percentage of dye removal (94.64\%) was achieved at $\mathrm{pH}$ 8. As mentioned above, the $\mathrm{pH}$ of the dye solution may affect the ionization of functional groups on the designed nanocomposite hydrogel adsorbent, which worked as the active sites of the hydrogel adsorbent, in turn, governing the electrostatic attraction between the anions on the hydrogel matrix and the cation on the MB dye [51,53, 58]. At acid conditions, the negative surface charges of the nanocomposite hydrogel adsorbent were reduced, which were undesirable for the removal of positively charged MB dye. At alkaline $\mathrm{pH} 8$, the surface negative charges of the hydrogel increased, which was favorable for the removal of $\mathrm{MB}$ due to the enhanced electrostatic attraction to the positively charged MB. Above $\mathrm{pH} 8$, the surface of the hydrogel exhibited negative electricity, which lessening the electrostatic attraction between $\mathrm{MB}$ and the hydrogel, consequently, causing smaller removal values $[55,58]$.

\subsubsection{Effect of Contact Time}

Contact time is a significant factor that indicates whether the adsorbent removes the target contaminant to reach the equilibrium [55]. The variation of MO removal rate and adsorption capacity over contact time were shown in Figure $7 \mathrm{~b}$. It was found that the adsorption capacity, as well as removal percentage, were improved with increasing immersion contact time. A fast adsorption for initial $60 \mathrm{~min}$ and relatively a slower adsorption after $60 \mathrm{~min}$ till $5 \mathrm{~h}$ were observed. At the same time, the color of the hydrogels became dark gradually, whereas the MB solution turned clear from an initial blue to completely transparent as shown in the inset of Figure $7 \mathrm{~b}$. These may be attributed to the filling of the all surface binding sites and diffusion of the MB molecules into pores of the designed beads. At the starting of adsorption process, there were plentiful active sites on the surface of the beads, which could combined with the MO molecules once they were added into dye solution [57, 58]. Moreover, the high MB concentration in the solution provided the apparent driving force for $\mathrm{MB}$ adsorption supported by concentration gradient. In the later stage of the adsorption process, where the concentration of $\mathrm{MB}$ decreased, the adsorption sites on the surface of the beads tended to be saturated. Therefore, the diffusion and adsorption of MB molecules deliberated, hence the adsorption rate decreased continuously [59]. 

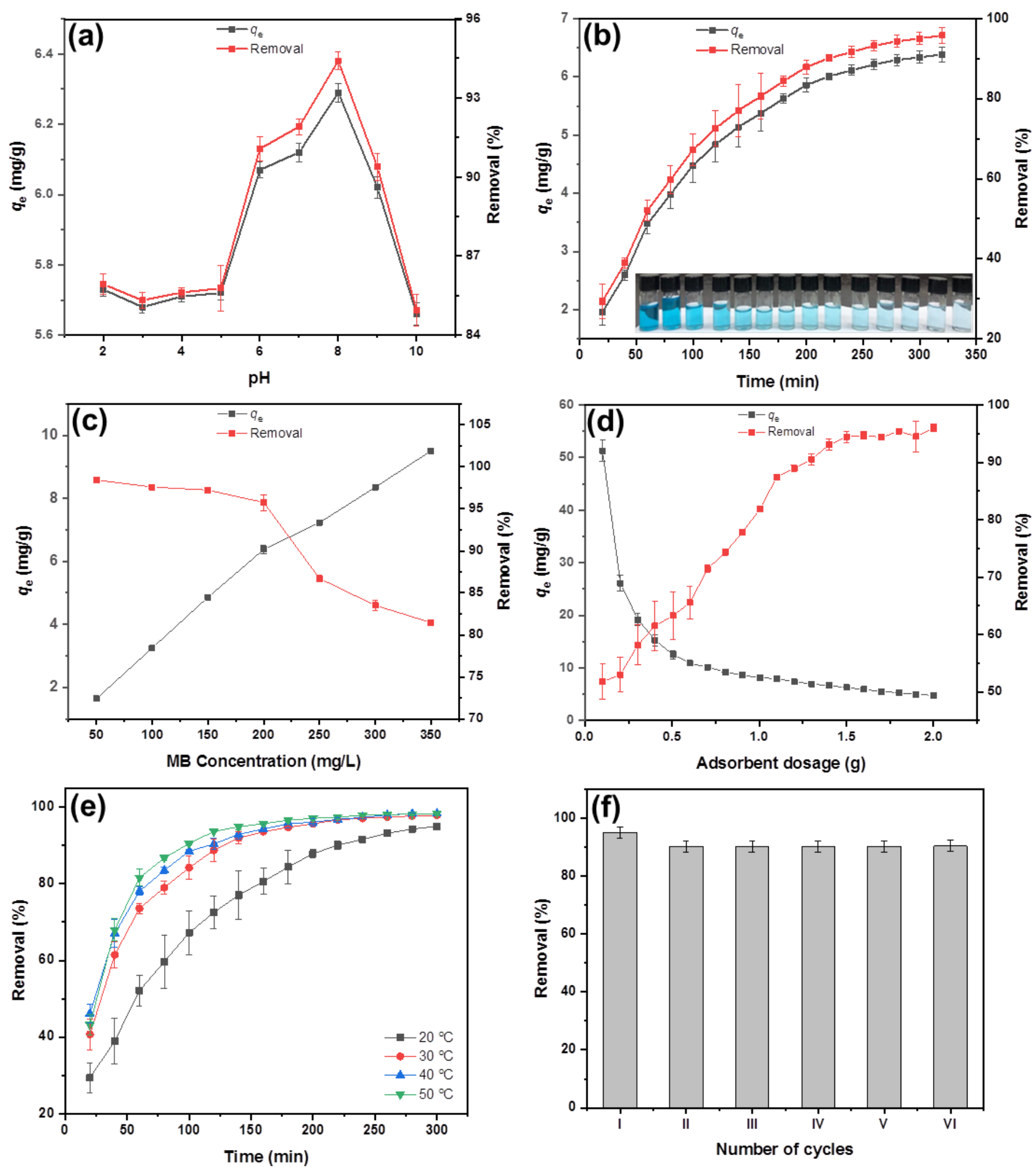

Figure 7. Effects of different factors on the adsorption capacity and removal percentage of MB onto the PVA-Alg/Bent nanocomposite hydrogel beads: (a) $\mathrm{pH}\left(\mathrm{C}_{\mathrm{o}}[\mathrm{MB}]=200 \mathrm{mg} / \mathrm{L}, \mathrm{V}=50 \mathrm{~mL}\right.$, beads dosage $=1.5 \mathrm{~g}, \mathrm{pH}=8, \mathrm{t}=300 \mathrm{~min}, \mathrm{~T}=$ $\left.30{ }^{\circ} \mathrm{C}\right),(\mathrm{b})$ contact time $\left(\mathrm{Co}[\mathrm{MB}]=200 \mathrm{mg} / \mathrm{L}, \mathrm{V}=50 \mathrm{~mL}, \mathrm{pH}=8\right.$, beads dosage $\left.=1.5 \mathrm{~g}, \mathrm{~T}=30{ }^{\circ} \mathrm{C}\right),(\mathrm{c}) \mathrm{MB}$ initial concentration $\left(\mathrm{V}=50 \mathrm{~mL}, \mathrm{pH}=8\right.$, beads dosage $\left.=1.5 \mathrm{~g}, \mathrm{t}=300 \mathrm{~min}, \mathrm{~T}=30{ }^{\circ} \mathrm{C}\right)$, $(\mathrm{d})$ adsorbent dosage $(\mathrm{Co}[\mathrm{MB}]=200$ $\left.\mathrm{mg} / \mathrm{L}, \mathrm{V}=50 \mathrm{~mL}, \mathrm{pH}=8, \mathrm{t}=300 \mathrm{~min}, \mathrm{~T}=30^{\circ} \mathrm{C}\right)$, (e) temperature $\left(\mathrm{C}_{\mathrm{o}}[\mathrm{MB}]=200 \mathrm{mg} / \mathrm{L}, \mathrm{V}=50 \mathrm{~mL}, \mathrm{pH}=8, \mathrm{beads}\right.$ dosage $=1.5 \mathrm{~g}, \mathrm{t}=300 \mathrm{~min}$.$) , and (\mathrm{f})$ reusability $\left(\mathrm{C}_{\mathrm{o}}[\mathrm{MB}]=200 \mathrm{mg} / \mathrm{L}, \mathrm{V}=50 \mathrm{~mL}, \mathrm{pH}=8\right.$, beads dosage $=1.5 \mathrm{~g}, \mathrm{t}=300 \mathrm{~min}, \mathrm{~T}=$ $\left.30{ }^{\circ} \mathrm{C}\right)$. 


\subsubsection{Effect of Initial MB Concentration}

In batch adsorption, the initial concentration of the $\mathrm{MB}$ molecules is the main driving force offsetting the mass diffusion resistance force [56]. Figure $7 \mathrm{c}$ shows the influence of the initial concentration of $\mathrm{MB}$ on adsorption capacity and removal percentage of MB. It could be noticed that the dye adsorption capacity increased with increasing initial $\mathrm{MB}$ concentration while the removal percentage declined. For instance, when the initial concentration of MB was 50,200 and $300 \mathrm{mg} / \mathrm{L}$, the removal rate of beads was about 97,85 and $69 \%$, respectively. This indicated that at a lower concentration of $\mathrm{MB}$, nanocomposite hydrogel beads had an abundance adsorption active sites leading to a high adsorption for the majority of MB molecules [60]. However, saturation of adsorption sites would be established with further increasing $\mathrm{MB}$ concentration, and adsorption became difficult since the increased numbers of MO molecules attached to the surface of the beads would repel free MO molecules [58,59, 60].

\subsubsection{Effect of Adsorbent Dosage}

Adsorbent dosage is another key factor in the dye adsorption system for maximizing the cost efficiency and benefits of adsorbents [60]. Figure $7 \mathrm{~d}$ illustrates the effects of adsorbent dosage on the MB adsorption capacity and removal percentage. When $0.1 \mathrm{~g}$ of the nanocomposite beads was applied, the MB adsorption capacity was 50 $\mathrm{mg} / \mathrm{g}$, constituting a removal percentage of $54 \%$. With increasing beads dosage above 0.1 $\mathrm{g}$, the adsorption capacity decreased, but the dye removal percentage increased gradually, eventually reaching an equilibrium at a maximum value of $94.64 \%$ on using beads dosage of $1.5 \mathrm{~g}$. This is because the concentration of dye in the solution was fixed, so excessive adsorbent dosage caused the active sites of the adsorbent to compete, resulting in a decrease in the amount of dye adsorbed per unit surface area [59,60]. Accordingly, the optimal beads dosage for MB removal was set as $1.5 \mathrm{~g}$.

\subsubsection{Effect of Temperature}

The influence of temperature on the removal rate is an important factor to investigate dye adsorption in industrial wastewater at different temperatures [55,58]. The dependence of the $\mathrm{MB}$ removal percentage on the temperature was examined at various temperatures ranging from $20-50{ }^{\circ} \mathrm{C}$ as presented in Figure 7e. Obviously, the removal percentage increased as the temperature rises from 20 to $30^{\circ} \mathrm{C}$. More specifically, the removal percentage reached $95 \%$ after $200 \mathrm{~min}$ at $30^{\circ} \mathrm{C}$, while it took $300 \mathrm{~min}$ to reach $90 \%$ at $20^{\circ} \mathrm{C}$. However, the removal percentage of $\mathrm{MB}$ at $40^{\circ} \mathrm{C}$ and $50^{\circ} \mathrm{C}$ revealed insignificant improvement compared with that at $30^{\circ} \mathrm{C}$. This finding was attributed to the increased mobility of the $\mathrm{MB}$ molecules at high temperature, which enhanced the MB molecules quickly get into the interior of hydrogel beads, resulting in an improvement of the removal percentage $[57,61]$. This behavior confirmed that the adsorption of dyes has an endothermic nature [61]. Hence, $30^{\circ} \mathrm{C}$ was chosen as the optimum solution temperature.

\subsubsection{Reusability of Adsorbent}

For acceptance of a system at industrial scale, the system should be economically feasible and repetitive. The reusability performance of adsorbent determines the feasibility of large-scale practical application [48, 49]. The prepared PVA-Alg/Bent (30 $\mathrm{wt} \%$ ) nanocomposite beads can be quickly separated from solution by simple separation technology, which provides the possibility for subsequent desorption and reuse. The nanocomposite beads for the dyes are reused for 6 cycles, which are washed by $0.1 \mathrm{M}$ $\mathrm{HCl}$ solution as the eluent. The reutilization was determined by sorption-desorption cycles of the beads. As shown in Figure 7f, the beads had good adsorption effect on MB after one cycle of adsorption-desorption tests with the removal percentage of $94.32 \%$. 
However, with the increase in the number of cycles, the removal percentage can be maintained at a steady value about $90 \%$, indicating that the adsorbent performed well in the structural stability [50]. The obtained results suggested that the PVA-Alg/Bent (30 $\mathrm{wt} \%$ ) nanocomposite beads have good application prospect because of the good adsorption performance, efficient reusability and low cost by using cheap, environment-friendly raw materials and simple manufacturing processes.

\subsection{Adsorption Isotherm Study}

The isotherm models of Langmuir and Freundlich are employed to get more insights into the removal process of MB dye by PVA-Alg/Bent nanocomposite hydrogel beads. The Langmuir isotherm model is assumed to have affinity for homogenous monolayer adsorption. While the Freundlich isotherm model is used to recognize the adsorption of multiple adsorption layer surfaces [50,51]. Figure 8 displays the adsorption isotherms of the MB removal by the nanocomposite hydrogel beads. In the present study, Langmuir and Freundlich models exposed a very high $\mathrm{R}^{2}$ value of 0.9932 and 0.9968 respectively, which indicated the suitability of both models to describe the adsorption of MB on hydrogel beads [61]. Accordingly, the adsorption process was likely to comprises both monolayer adsorption on homogeneous surface and multilayer adsorption on heterogeneous surface. The results in Table 1 shown that, RL values lay between 0.005 to $0.03\left(0<\mathrm{R}_{\mathrm{L}}<1\right)$ and $1 / \mathrm{n}$ was about $0.57(0<1 / \mathrm{n}<1)$ as well as $q_{m}$ of $51.34 \mathrm{mg} / \mathrm{g}$ that confirmed the feasibility and favorability of the MB adsorption on the surface of the beads.
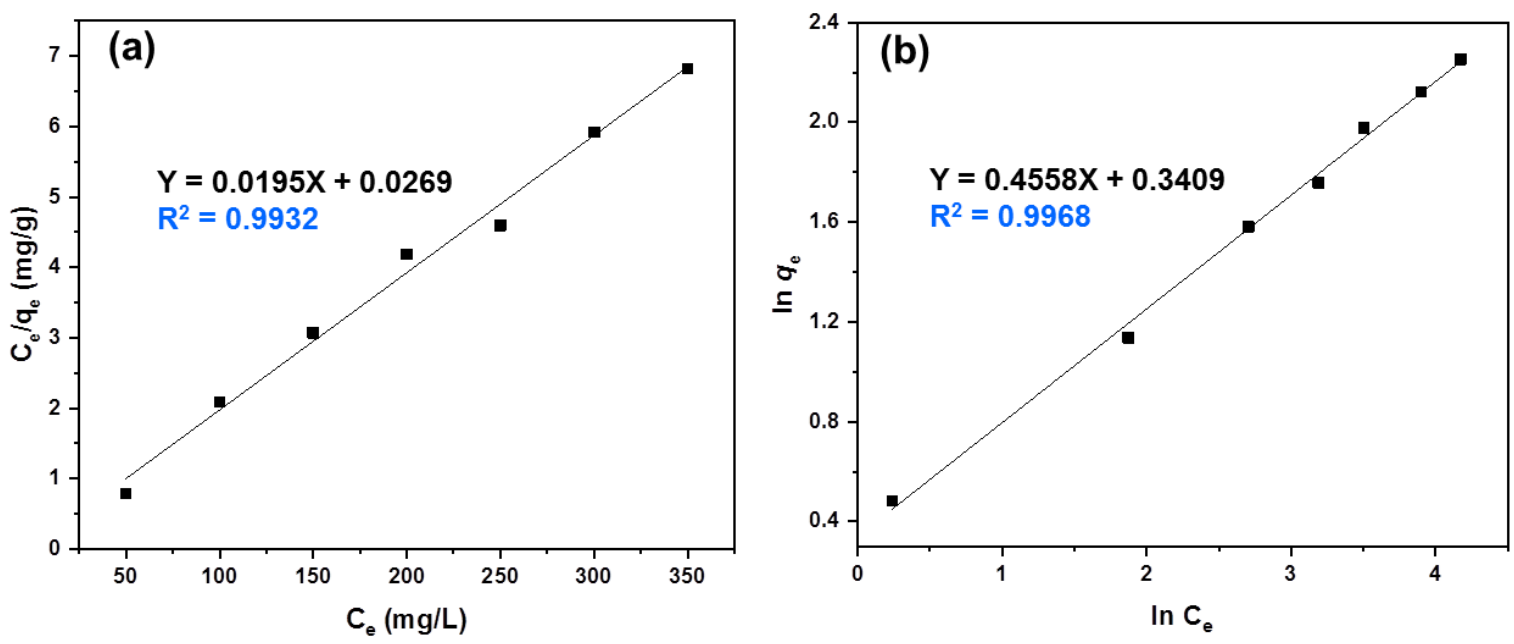

Figure 8. (a) The Langmuir adsorption isotherm and (b) Freundlich adsorption isotherm, for the adsorption of the MB onto the PVA-Alg/Bent nanocomposite hydrogel beads.

\subsection{Adsorption Kinetics Study}

The kinetics of adsorption are an important index in defining the efficiency of sorption [58]. In this study, the kinetic behavior of MB sorption onto the beads was investigated using both the pseudo-first-order and pseudo-second-order kinetic models. Linear plots of $\ln \left(q_{e}-q_{t}\right)$ and $t / q_{t}$ versus contact time are shown in Figures $9 a$ and $9 b$ respectively. It can be seen that the $\mathrm{R}^{2}$ value $(0.9974)$ of the pseudo-second-order kinetic model was higher than that of the pseudo-first-order kinetic model (0.9615). Hence, the pseudo-second-order kinetic model is more fitting for describing the adsorption behavior of $\mathrm{MB}$ onto beads, and the main adsorption process of $\mathrm{MB}$ was dominated by chemisorption mechanism. 

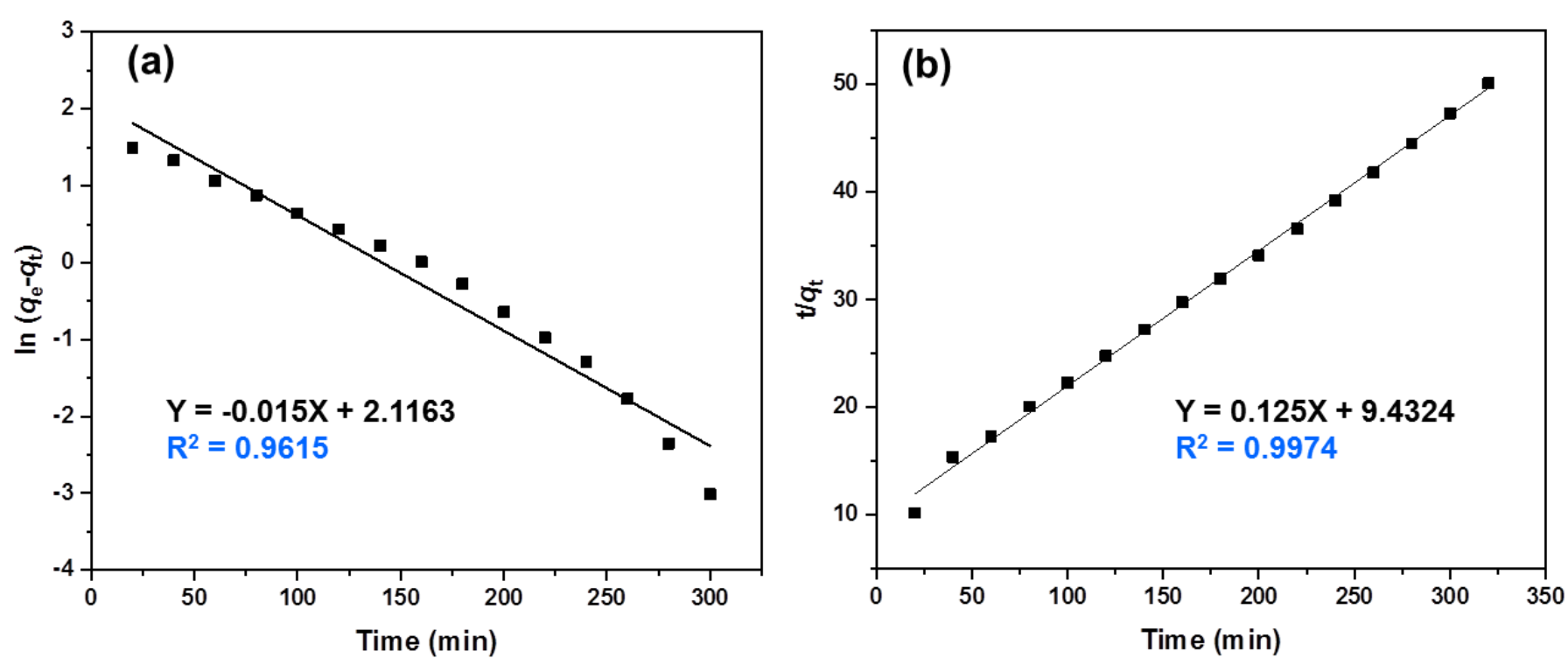

Figure 9. (a) The pseudo-first-order and (b) pseudo-second-order kinetic models for the adsorption of the MB onto the PVA-Alg/Bent nanocomposite hydrogel beads.

\subsection{Adsorption Mechanism of Cationic MB Dye}

Figure 10 displays an illustrative scheme for the proposed adsorption mechanism. As mentioned before, in alkaline $\mathrm{pH}$; the surface negative charge of the PVA-Alg/Bent nanocomposite hydrogel gel adsorbent increased due to the ionization of $-\mathrm{COOH}$ and $\mathrm{SiOH}$ groups into $-\mathrm{COO}^{-}$and $-\mathrm{SiO}^{-}$respectively. Thus, the removal of $\mathrm{MB}$ by the designed adsorbent is due to the electrostatic interactions between the positive charges on iminium groups $(=\mathrm{N}+)$ present in the $\mathrm{MB}$ dye molecules and the abundant negative charges of the nanocomposite adsorbent. In addition, hydrogen bonding occurs between the $-\mathrm{OH}$ groups present in the adsorbent surface and nitrogen atom of $\mathrm{MB}[61,62]$. 


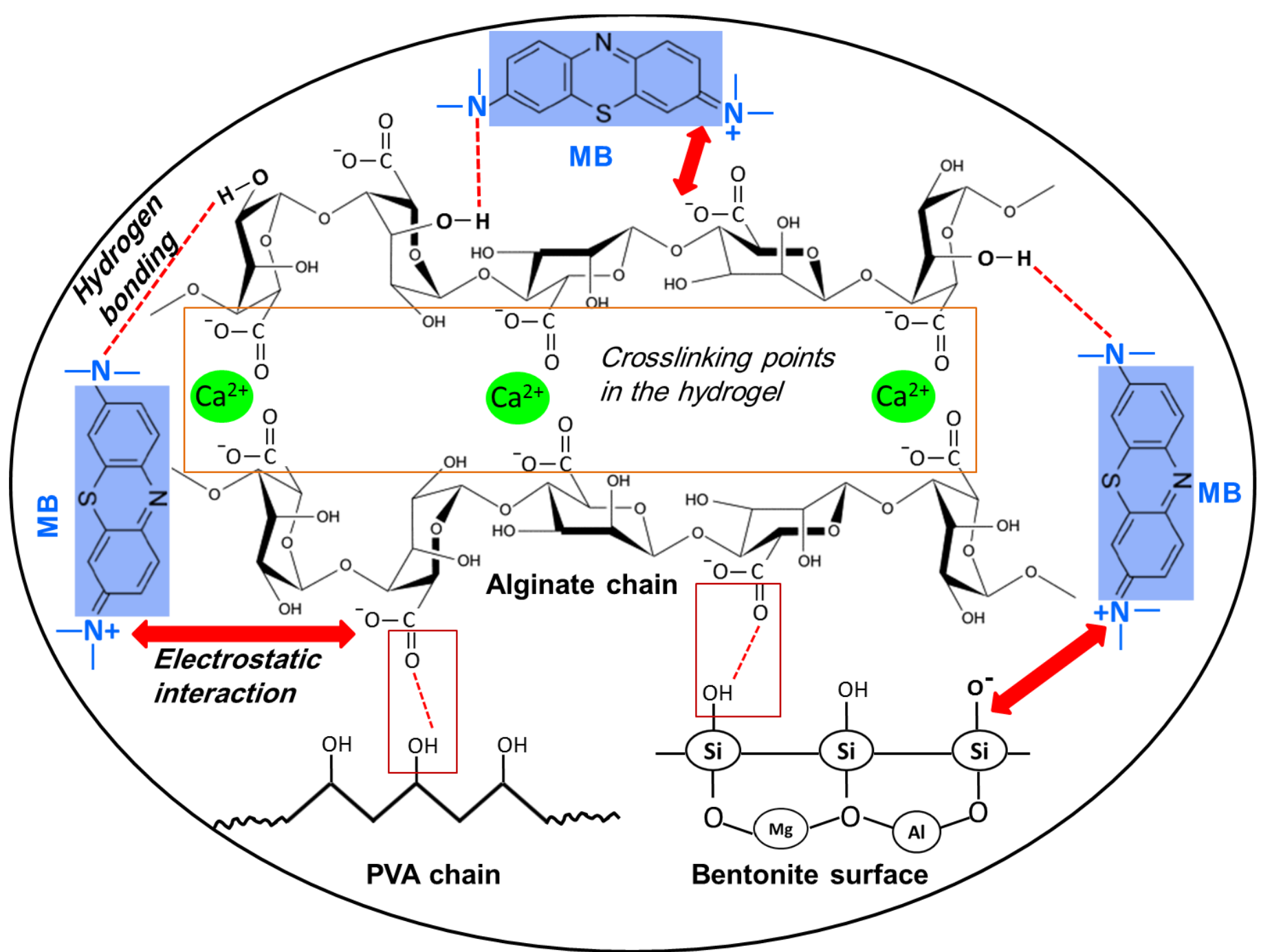

Figure 10. Proposed scheme indicating the possible interaction mechanism of the MB onto the PVA-Alg/Bent nanocomposite hydrogel beads

\section{Conclusion}

In the present study, PVA-Alg/Bent nanocomposite hydrogel was successfully developed as efficient adsorbent for removal of MB from aqueous solution. The designed adsorbent possessed porous structure, high specific surface area, and net negative surface charge provided many channels for the rapid adsorption of MB dye. Adsorbent beads had a good stability and reusability after six repeated cycles of adsorption and desorption. Moreover, the adsorbent showed outstanding MB removal efficiency with easy handling and extraction after adsorption, as well as eco-friendly and low cost. Therefore, It could be suitable for the removal of other toxic cationic dyes and harmful pollutants from wastewater at industrial scale.

Author Contributions: Conceptualization, A.A.E.-F.; Methodology, A.A.E.-F. and M.A.A.A.; Data analysis and interpretations, A.A.E.-F. and M.A.A.A.; Supervision, A.A.E.-F. and S.R.; Writing-original draft preparation, M.A.A.A.; Writing-review \& editing, A.A.E.-F. and S.R. All authors have read and agreed to the published version of the manuscript.

Funding: This research received no external funding.

Data Availability Statement: Not applicable 
Conflicts of Interest: The authors declare no conflict of interest.

\section{References}

1. Eltaweil, A.S.; Mamdouh, I.M.; Abd El-Monaem, E.M.; El-Subruiti, G.M. Highly efficient removal for methylene blue and Cu ${ }^{2+}$ onto UiO-66 metal-organic framework/carboxylated graphene oxide-incorporated sodium alginate beads. ACS omega, 2021, 6, 23528-23541.

2. Liu, X.J.; Li, M.F.; Singh, S.K. Manganese-modified lignin biochar as adsorbent for removal of methylene blue. Journal of Materials Research and Technology, 2021, 12, 1434-1445.

3. Olusegun, S.J.; Freitas, E.T.; Lara, L.R.; Mohallem, N.D. Synergistic effect of a spinel ferrite on the adsorption capacity of nano bio-silica for the removal of methylene blue. Environmental technology, 2021, 42, 2163-2176.

4. Shen, Y.; Ni, W.X.; Li, B. Porous organic polymer synthesized by green diazo-coupling reaction for adsorptive removal of methylene blue. ACS omega, 2021, 6, 3202-3208.

5. Silvestri, S.; Stefanello, N.; Sulkovski, A.A.; Foletto, E.L. Preparation of $\mathrm{TiO}_{2}$ supported on MDF biochar for simultaneous removal of methylene blue by adsorption and photocatalysis. Journal of Chemical Technology E Biotechnology, 2020, 95, $2723-2729$.

6. Shrestha, P.; Jha, M.K.; Ghimire, J.; Koirala, A.R.; Shrestha, R.M.; Sharma, R.K.; Pant, B.; Park, M.; Pant, H.R. Decoration of zinc oxide nanorods into the surface of activated carbon obtained from agricultural waste for effective removal of methylene blue dye. Materials, 2020,13, 5667.

7. Arias Arias, F.; Guevara, M.; Tene, T.; Angamarca, P.; Molina, R.; Valarezo, A.; Salguero, O.; Vacacela Gomez, C.; Arias, M.; Caputi, L.S. The adsorption of methylene blue on eco-friendly reduced graphene oxide. Nanomaterials, 2020, $10,681$.

8. Nayl, A.A.; Abd-Elhamid, A.I.; Abu-Saied, M.A.; El-Shanshory, A.A.; Soliman, H.M.; Akl, M.A; Aly, H.F. A novel method for highly effective removal and determination of binary cationic dyes in aqueous media using a cotton-graphene oxide composite. RSC Advances, 2020, 10, 7791-7802.

9. Duhan, M.; Kaur, R. Nano-structured polyaniline as a potential adsorbent for methylene blue dye removal from effluent. Composite Science and Technology, 2021, 5, 7.

10. Alharthi, F.A.; Ali Alghamdi, A.; Alanazi, H.S.; Alsyahi, A.A.; Ahmad, N. Photocatalytic Degradation of the Light Sensitive Organic Dyes: Methylene Blue and Rose Bengal by Using Urea Derived g-C3N4/ZnO Nanocomposites. Catalysts, 2020, $10,1457$.

11. Palani, G.; Arputhalatha, A.; Kannan, K.; Lakkaboyana, S.K.; Hanafiah, M.M.; Kumar, V.; Marella, R.K. Current trends in the application of nanomaterials for the removal of pollutants from industrial wastewater treatment-a review. Molecules, 2021, 26, 2799.

12. Norrrahim, M.N.F.; Kasim, N.A.M.; Knight, V.F.;Misenan, M.S.M.; Janudin, N.; Shah, N.A.A.; Kasim, N.; Yusoff, W.Y.W.; Noor, S.A.M.; Jamal, S.H; Ong, K.K. Nanocellulose: a bioadsorbent for chemical contaminant remediation. RSC Advances, 2021, 11, 7347-7368.

13. Mon, M.; Bruno, R.; Ferrando-Soria, J.; Armentano, D.; Pardo, E. Metal-organic framework technologies for water remediation: towards a sustainable ecosystem. Journal of materials chemistry A, 2018, 6, 4912-4947.

14. Punia, P.; Bharti, M.K.; Chalia, S.; Dhar, R.; Ravelo, B.; Thakur, P.; Thakur, A. Recent advances in synthesis, characterization, and applications of nanoparticles for contaminated water treatment-a review. Ceramics International, 2021, 47, 1526-1550.

15. Taneez, M.; Hurel, C. A review on the potential uses of red mud as amendment for pollution control in environmental media. Environmental Science and Pollution Research, 2019, 26, 22106-22125.

16. Wang, S.; Li, X.; Liu, Y.; Zhang, C.; Tan, X.; Zeng, G.; Song, B.; Jiang, L. Nitrogen-containing amino compounds functionalized graphene oxide: synthesis, characterization and application for the removal of pollutants from wastewater: a review. Journal of hazardous materials, 2018, 342, 177-191.

17. Rasool, K.; Pandey, R.P.; Rasheed, P.A.; Buczek, S.; Gogotsi, Y.; Mahmoud, K.A. Water treatment and environmental remediation applications of two-dimensional metal carbides (MXenes). Materials Today, 2019, 30, 80-102. 
18. Chen, Y.; Lin, Z.; Hao, R.; Xu, H.; Huang, C. Rapid adsorption and reductive degradation of Naphthol Green B from aqueous solution by Polypyrrole/Attapulgite composites supported nanoscale zero-valent iron. Journal of hazardous materials, 2019, 371, 8-17.

19. Garba, Z.N.; Zhou, W.; Lawan, I.; Xiao, W.; Zhang, M.; Wang, L.; Chen, L.; Yuan, Z. An overview of chlorophenols as contaminants and their removal from wastewater by adsorption: A review. Journal of environmental management, 2019, 241, 59-75.

20. Tang, H.; Zhao, Y.; Shan, S.; Yang, X.; Liu, D.; Cui, F.; Xing, B. Theoretical insight into the adsorption of aromatic compounds on graphene oxide. Environmental Science: Nano, 2018, 5, 2357-2367.

21. Wang, H.; Xu, J.; Liu, X.; Sheng, L. Preparation of straw activated carbon and its application in wastewater treatment: a review. Journal of Cleaner Production, 2021, 283, 124671.

22. Bharath, G.; Hai, A.; Rambabu, K.; Savariraj, D.; Ibrahim, Y.; Banat, F. The fabrication of activated carbon and metal-carbide 2D framework-based asymmetric electrodes for the capacitive deionization of $\mathrm{Cr}$ (vi) ions toward industrial wastewater remediation. Environmental Science: Water Research E Technology, 2020, 6, 351-361.

23. Mouiya, M.; Bouazizi, A.; Abourriche, A.; Benhammou, A.; El Hafiane, Y.; Ouammou, M.; Abouliatim, Y.; Younssi, S.A.; Smith, A.; Hannache, H. Fabrication and characterization of a ceramic membrane from clay and banana peel powder: application to industrial wastewater treatment. Materials Chemistry and Physics, 2019, 227, 291-301.

24. Adam, M.R.; Salleh, N.M.; Othman, M.H.D.; Matsuura, T.; Ali, M.H.; Puteh, M.H.; Ismail, A.F.; Rahman, M.A.; Jaafar, J. The adsorptive removal of chromium (VI) in aqueous solution by novel natural zeolite based hollow fibre ceramic membrane. Journal of environmental management, 2018, 224, 252-262.

25. Nagpal, M.; Kakkar, R. Use of metal oxides for the adsorptive removal of toxic organic pollutants. Separation and Purification Technology, 2019, 211, 522-539.

26. Crini, G.; Torri, G.; Lichtfouse, E.; Kyzas, G.Z.; Wilson, L.D.; Morin-Crini, N. Dye removal by biosorption using cross-linked chitosan-based hydrogels. Environmental Chemistry Letters, 2019, 17, 1645-1666.

27. Pakdel, P.M.; Peighambardoust, S.J. Review on recent progress in chitosan-based hydrogels for wastewater treatment application. Carbohydrate polymers, 2018, 201, 264-279.

28. Van Tran, V.; Park, D.; Lee, Y.C. Hydrogel applications for adsorption of contaminants in water and wastewater treatment. Environmental Science and Pollution Research, 2018, 25, 24569-24599.

29. Bandara, P.C.; Perez, J.V.D.; Nadres, E.T.; Nannapaneni, R.G.; Krakowiak, K.J.; Rodrigues, D.F. Graphene oxide nanocomposite hydrogel beads for removal of selenium in contaminated water. ACS Applied Polymer Materials, 2019, 1, 2668-2679.

30. Horn, C.; Pospiech, D.; Allertz, P.J.; Muiller, M.; Salchert, K.; Hommel, R. Chemical Design of Hydrogels with Immobilized Laccase for the Reduction of Persistent Trace Compounds in Wastewater. ACS Applied Polymer Materials, 2021, 3, $2823-2834$.

31. Sadegh, H.; Ali, G.A.; Gupta, V.K.; Makhlouf, A.S.H.; Shahryari-Ghoshekandi, R.; Nadagouda, M.N.; Sillanpää, M.; Megiel, E. The role of nanomaterials as effective adsorbents and their applications in wastewater treatment. Journal of Nanostructure in Chemistry, 2017, 7, 1-14.

32. Shalla, A.H.; Yaseen, Z.; Bhat, M.A.; Rangreez, T.A.; Maswal, M. Recent review for removal of metal ions by hydrogels. Separation Science and Technology, 2019, 54, 89-100.

33. Elbedwehy, A.M.; Atta, A.M. Novel superadsorbent highly porous hydrogel based on arabic gum and acrylamide grafts for fast and efficient methylene blue removal. Polymers, 2020, 12, 338.

34. Wang, N.; Xiao, W.; Niu, B.; Duan, W.; Zhou, L.; Zheng, Y.. Highly efficient adsorption of fluoroquinolone antibiotics using chitosan derived granular hydrogel with 3D structure. Journal of Molecular Liquids, 2019, 281, 307-314.

35. Fan, X.M.; Yu, H.Y.; Wang, D.C.; Mao, Z.H.; Yao, J.; Tam, K.C. Facile and green synthesis of carboxylated cellulose nanocrystals as efficient adsorbents in wastewater treatments. ACS Sustainable Chemistry \& Engineering, 2019, 7, 18067-18075. 
36. Erfani, M.; Javanbakht, V. Methylene Blue removal from aqueous solution by a biocomposite synthesized from sodium alginate and wastes of oil extraction from almond peanut. International journal of biological macromolecules, 2018, 114, $244-255$.

37. Mokhena, T.C.; Jacobs, N.V.; Luyt, A.S. Nanofibrous alginate membrane coated with cellulose nanowhiskers for water purification. Cellulose, 2018, 25, 417-427.

38. Luo, H.; Zeng, X.; Liao, P.; Rong, H.; Zhang, T.C.; Zhang, Z.J.; Meng, X. Phosphorus removal and recovery from water with macroporous bead adsorbent constituted of alginate- $\mathrm{Zr}^{4+}$ and PNIPAM-interpenetrated networks. International journal of biological macromolecules, 2019, 126,1133-1144.

39. Mok, C.F.; Ching, Y.C.; Muhamad, F.; Osman, N.A.A.; Dai Hai, N.; Hassan, C.R.C. Adsorption of dyes using poly (vinyl alcohol)(PVA) and PVA-based polymer composite adsorbents: a review. Journal of Polymers and the Environment, 2020, 28, 775-793.

40. Ali, I.; Peng, C.; Naz, I.; Lin, D.; Saroj, D.P.; Ali, M. Development and application of novel bio-magnetic membrane capsules for the removal of the cationic dye malachite green in wastewater treatment. RSC advances, 2019, 9, 3625-3646.

41. Cavallaro, G.; Lazzara, G.; Rozhina, E.; Konnova, S.; Kryuchkova, M.; Khaertdinov, N.; Fakhrullin, R. Organic-nanoclay composite materials as removal agents for environmental decontamination. RSC Advances, 2019, 9, 40553-40564.

42. Hosseini, S.A.; Daneshvar e Asl, S.; Vossoughi, M.; Simchi, A.; Sadrzadeh, M. Green Electrospun Membranes Based on Chitosan/Amino-Functionalized Nanoclay Composite Fibers for Cationic Dye Removal: Synthesis and Kinetic Studies. ACS omega, 2021, 6,10816-10827.

43. Fernandes, J.V.; Rodrigues, A.M.; Menezes, R.R.; Neves, G.D.A. Adsorption of anionic dye on the acid-functionalized bentonite. Materials, 2020, 13, 3600 .

44. Baigorria, E.; Cano, L.A.; Sanchez, L.M.; Alvarez, V.A.; Ollier, R.P. Bentonite-composite polyvinyl alcohol/alginate hydrogel beads: Preparation, characterization and their use as arsenic removal devices. Environmental Nanotechnology, Monitoring $\mathcal{E}$ Management, 2020, 14,100364.

45. Zvulunov, Y.; Radian, A. Alginate Composites Reinforced with Polyelectrolytes and Clay for Improved Adsorption and Bioremediation of Formaldehyde from Water. ACS ESET Water, 2021,1,1837-1848.

46. Abd El-Fattah, A.; Mansour, A. Viscoelasticity, mechanical properties, and in vitro biodegradation of injectable chitosan-poly (3-hydroxybutyrate-co-3-hydroxyvalerate)/nanohydroxyapatite composite hydrogel. Bulletin of Materials Science. 2018, 41, 1-10.

47. Asadi, S.; Eris, S.; Azizian, S. Alginate-based hydrogel beads as a biocompatible and efficient adsorbent for dye removal from aqueous solutions. ACS omega, 2018, 3, 15140-15148.

48. Hong, G.B.; Yu, T.J.; Lee, H.C.; Ma, C.M. Using Rice Bran Hydrogel Beads to Remove Dye from Aqueous Solutions. Sustainability, 2021, 13, 5640.

49. Yadav, S.; Asthana, A.; Chakraborty, R.; Jain, B.; Singh, A.K.; Carabineiro, S.A.; Susan, M.; Hasan, A.B. Cationic dye removal using novel magnetic/activated charcoal/ $\beta$-cyclodextrin/alginate polymer nanocomposite. Nanomaterials, 2020, 10,170.

50. Pandey, L.M. Enhanced adsorption capacity of designed bentonite and alginate beads for the effective removal of methylene blue. Applied Clay Science, 2019, 169, 102-111.

51. Qi, X.; Wu, L.; Su, T.; Zhang, J.; Dong, W. Polysaccharide-based cationic hydrogels for dye adsorption. Colloids and Surfaces B: Biointerfaces, 2018, 170, 364-372.

52. Merakchi, A.; Bettayeb, S.; Drouiche, N.; Adour, L.; Lounici, H. Cross-linking and modification of sodium alginate biopolymer for dye removal in aqueous solution. Polymer Bulletin, 2019, 76, 3535-3554.

53. Oussalah, A.; Boukerroui, A. Alginate-bentonite beads for efficient adsorption of methylene blue dye. Euro-Mediterranean Journal for Environmental Integration, 2020, 5, 1-10.

54. Wang, W.; Zhao, Y.; Bai, H.; Zhang, T.; Ibarra-Galvan, V.; Song, S. Methylene blue removal from water using the hydrogel beads of poly (vinyl alcohol)-sodium alginate-chitosan-montmorillonite. Carbohydrate polymers, 2018, 198, 518-528. 
55. Wu, N.; Niu, F.; Lang, W.; Xia, M. Highly efficient flame-retardant and low-smoke-toxicity poly (vinyl alcohol)/alginate/montmorillonite composite aerogels by two-step crosslinking strategy. Carbohydrate polymers, 2019, 221, 221-230.

56. da Silva Fernandes, R.; de Moura, M.R.; Glenn, G.M.; Aouada, F.A. Thermal, microstructural, and spectroscopic analysis of $\mathrm{Ca}^{2+}$ alginate/clay nanocomposite hydrogel beads. Journal of Molecular Liquids, 2018, 265, 327-336.

57. Hu, T.; Liu, Q.; Gao, T.; Dong, K.; Wei, G.; Yao, J. Facile preparation of tannic acid-poly (vinyl alcohol)/sodium alginate hydrogel beads for methylene blue removal from simulated solution. ACS omega, 2018, 3, 7523-7531.

58. Hou, N.; Wang, R.; Wang, F.; Bai, J.; Zhou, J.; Zhang, L.; Hu, J.; Liu, S.; Jiao, T. Fabrication of Hydrogels via Host-Guest Polymers as Highly Efficient Organic Dye Adsorbents for Wastewater Treatment. ACS omega, 2020, 5, 5470-5479.

59. Belhouchat, N.; Zaghouane-Boudiaf, H.; Viseras, C. Removal of anionic and cationic dyes from aqueous solution with activated organo-bentonite/sodium alginate encapsulated beads. Applied Clay Science, 2017, 135, 9-15.

60. Su, T.; Wu, L.; Pan, X.; Zhang, C.; Shi, M.; Gao, R.; Qi, X.; Dong, W. Pullulan-derived nanocomposite hydrogels for wastewater remediation: Synthesis and characterization. Journal of colloid and interface science, 2019, 542, 253-262.

61. Zhang, Z.H.; Xu, J.Y.; Yang, X.L. MXene/sodium alginate gel beads for adsorption of methylene blue. Materials Chemistry and Physics, 2021, 260, 124123.

62. Jana, S.; Ray, J.; Mondal, B.; Tripathy, T. Efficient and selective removal of cationic organic dyes from their aqueous solutions by a nanocomposite hydrogel, katira gum-cl-poly (acrylic acid-co-N, N-dimethylacrylamide)@bentonite. Applied Clay Science, 2019, 173, 46-64. 